\title{
A Mathematical Model of Shifted Gamma Distribution for Internet Traffic Packet Delay over a Next Generation Core Internetwork
}

\author{
Dao Ngoc Lam ${ }^{1)}$, Le Huu Lap ${ }^{2)}$, Le Nhat Thang ${ }^{2)}$ \\ ${ }^{1)}$ School Vietnam Telecom National Company,
Vietnam Posts and Telecommunications Group, Danang, Vietnam, lamdn.vtn3@vnpt.vn \\ ${ }^{2)}$ Posts and Telecom Institute of Technology \\ Vietnam Posts and Telecommunications Group, Hanoi, Vietnam \\ E-mail: 1amdn.vtn3@vnpt.vn, laplh@ptit.edu.vn, thangln@ptit.edu.vn
}

Abstract: Broadband Internet traffic is transported over the next generation core internetworks, which are composed of several IP/MPLS/GE network sections and transport multi-services. In practice, IP packet delay is normally measured in each separated network section but not over a whole internetwork. It is proved in the paper that packet delay distribution of Internet traffic component in core network sections can be approximately expressed as a shifted gamma distribution. Moreover a new explicit mathematical model based on shifted gamma distribution has also been proposed to compose delay distribution of Internet traffic packet transported over a core internetwork from component ones in each network section. It is resulted from this model that Internet packet delay over an internetwork inherits distribution properties from that over component networks. Other properties and parameters relationship of the model such as additive property of shape and location parameters, the relation between distribution lower moments and parameters, the dependence of distribution on parameters variation are also exposed in the paper. The proposed model of IP packet delay distributions has a certain scientific significance and plays an important role in practical performance analysis, network planning, designing and traffic engineering for improving the quality of service.

\section{INTRODUCTION}

Broadband or high speed Internet traffic is transported in the form of Internet Protocol (IP) packets over the next generation core networks which are based on multi-service platform and advanced technologies such as multi-protocol label switching (MPLS), multi (up to 100) Gigabit Ethernet (GE) and optical transport network (OTN). IP packet delay is one of thekey performance metrics of the next generation transport networks. IP packet delay distribution can be resulted from direct measurements [1], [2] or by estimating moments of the total delay distribution from component ones [3]. However, the first method is difficult to be implemented in case of multi-domain networks due to the management policies from different network providers while the second one has limited accuracy due to approximations and probability distribution of IP packet delay is not completely quantified. Moreover, IP packets can be routed and forwarded through different network sections where IP delay distributions are normally predetermined. Therefore, a method for composing internetwork delay distribution from component ones is necessary for flexible calculations of network performance and minimizing network operating and maintenance costs.

The previous research results [4],[5], [6], [7] show that in certain cases, end-to-end delay of IP packets in a fixed path over a traditional internet network can be approximately representedby a shifted (along the time axis)gamma distribution or typically possessesa gamma-like distribution with observed parametersdepend onlink capacity, node processing features, traffic and load conditions, network segments and paths (e.g., regional, national backbone, cross-country) and the time of day, etc. However, the IP packet delay distributions have not been represented adequately in form of mathematical models yet. Moreover, mathematical model of composed delay distribution over an internetwork and its parameter relationship with component ones have not been mentioned. 
Research, Development and Application on Information and Communication Technology

As an approach to solve the limitations mentioned above, it is proved in the paper that one-way delay distribution of IP packets transported over an internetwork (hereinafter referred to as composed distribution) can be composed from their delay distributions innetwork sections (also referred to as component distributions) by explicit mathematical models based on the shifted gamma distribution with certain assumptions. Moreover, the relationship of distribution parameters isalso considered on variant forms of IP packet delay distributions.

The remainder is organized as follows. A mathematical method used for composing delay distributions of IP packets from component ones is introduced in the part II. This method is applied to compose a distribution model of Internet traffic packet delay over a next generation internetwork with shifted gamma distribution for IP packet delay in each component network based on empirical results introduced in the part III. Further, the analyses and comments on results and parameter relationships are discussed in the part IV. Finally, the conclusion isincluded in the part V.

\section{A METHOD OF COMPOSING DELAY DISTRIBUTIONS}

An internetwork path consisting of $N$ consecutive network sections is considered in the paper as described in Fig. 1.

It is assumed that random variables of IP packet delay in $N$ network sections are mutually independent and their probability distributions can be represented by explicit probability density functions as called $P D F_{i}(t)$. According to probability theory [8] and additive property of IP packet delay, probability density function of IP packet delay in the whole internetwork, $P D F_{\Sigma}(t)$, can be composed by a convolution $(*)$ as follows:

$$
P D F_{\Sigma}(t)=\left(P D F_{1} * P D F_{2} * \ldots * P D F_{N}\right)(t) .
$$

It can be resulted from (1) as follows:

$$
\begin{array}{ll} 
& \mathcal{L}\left\{P D F_{\Sigma}(t)\right\}=\prod_{i=1}^{N} \mathcal{L}\left\{P D F_{i}(t)\right\} \\
\text { or } & P D F_{\Sigma}(t)=\mathcal{L}^{-1}\left\{\prod_{i=1}^{N} \mathcal{L}\left[P D F_{i}(t)\right]\right\}
\end{array}
$$

The Laplace-Stieltjestransform $\left(\mathcal{L}^{*}\right)$ [9] of cumulative distribution function of IP packet delay in an internetwork, as called $C D F_{\Sigma}(t)$, can be written as (4):

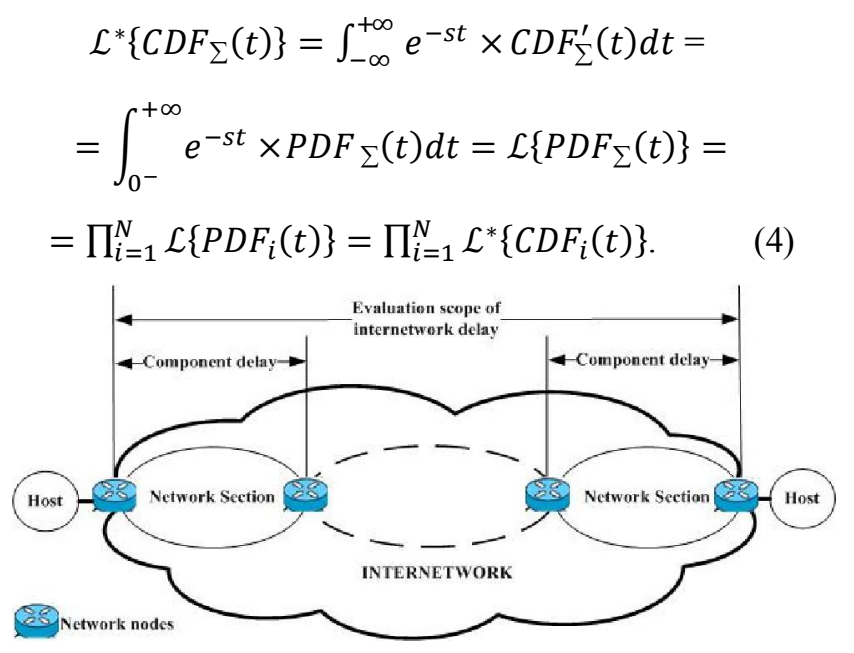

(a) Network scope of IP packet delay

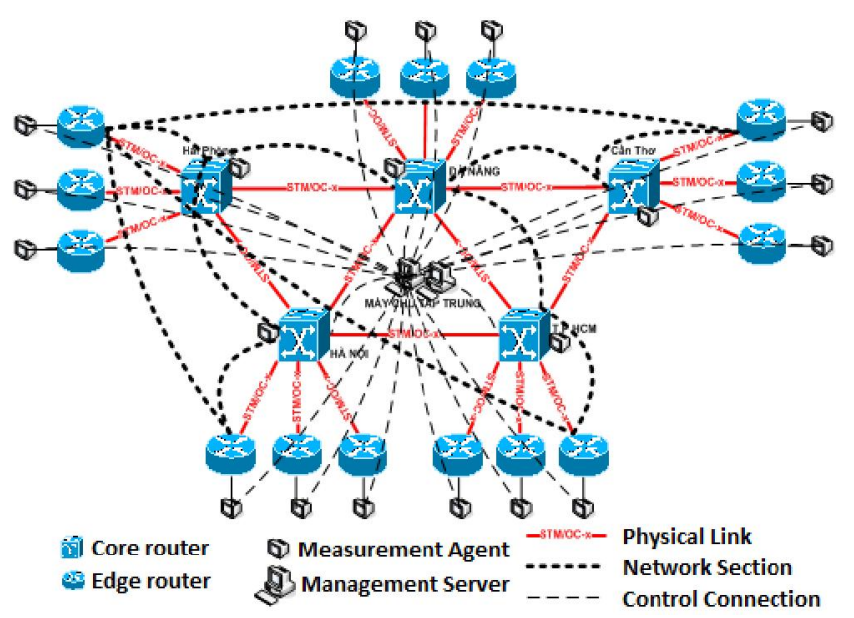

(b) VNPT next generation IP core network

Figure 1. Experimental scenario for IP packet delay measurement

Applying the Laplace-Stieltjes transform $\left(\mathcal{L}^{*}\right)^{-1}$ [9] on (4) results in (5):

$$
C D F_{\Sigma}(t)=\left(\mathcal{L}^{*}\right)^{-1}\left\{\prod_{i=1}^{N} \mathcal{L}^{*}\left[C D F_{i}(x)\right]\right\}
$$

or it can be alternatively calculated from (2) as follows:

where $\mathcal{L}^{-1}$ is the inverse Laplace transform. 


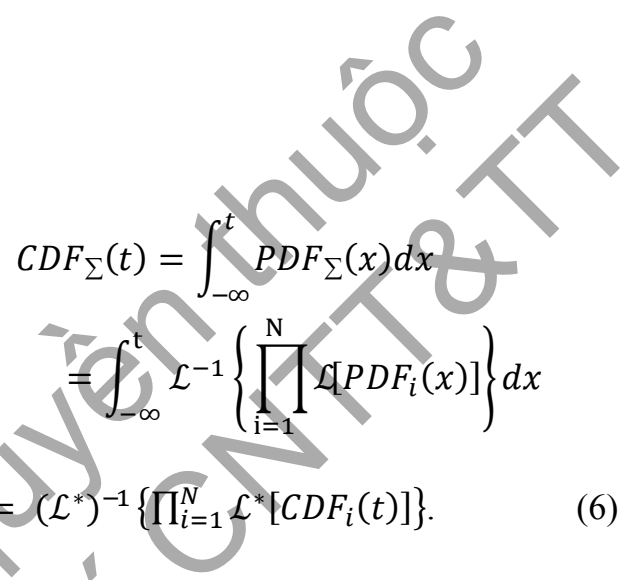

III. A MATHEMATICAL MODEL OF COMPOSED SHIFTED GAMMADISTRIBUTION FOR IP PACKET DELAY

A. A General Model

IP packet delay in each network section is supposed to conform to a shifted gamma distribution with the probability density function of $P D F_{i}(t)$ and the cumulative distribution function of $C D F_{i}(t)$ for the $i^{\text {th }}$ network section as follows:

$$
P D F_{i}(t)=\frac{\left[t-l_{i}\right]^{\left(k_{i}-1\right)} \times e^{-\frac{\left(t-l_{i}\right)}{\theta_{i}}}}{\theta_{i}^{k_{i}} \times \Gamma\left(k_{i}\right)} \times u\left(t-l_{i}\right),
$$

in which $\Gamma(k)=\int_{0}^{\infty} x^{(k-1)} e^{-x} d x$ is the gamma function;

$$
\begin{gathered}
C D F_{i}(t)=\int_{-\infty}^{t} P D F_{i}(x) d x= \\
\int_{l_{i}}^{t} \frac{\left[x-l_{i}\right]^{\left(k_{i}-1\right)} \times e^{-\frac{\left(x-l_{i}\right)}{\theta_{i}}}}{\theta_{i}^{k_{i}} \times \Gamma\left(k_{i}\right)} d x= \\
=\frac{1}{\Gamma\left(k_{i}\right)} \times \int_{l_{i}}^{t}\left[\left(\frac{x-l_{i}}{\theta_{i}}\right)^{\left(k_{i}-1\right)} \times e^{\left.-\left(\frac{x-l_{i}}{\theta_{i}}\right)\right] d\left(\frac{x-l_{i}}{\theta_{i}}\right)=}\right. \\
=\frac{1}{\Gamma\left(k_{i}\right)} \times \int_{0}^{\left(\frac{t-l_{i}}{\theta_{i}}\right)}\left[y^{\left(k_{i}-1\right)} \times e^{-y}\right] d y=\frac{\gamma\left(k_{i}, \frac{t-l_{i}}{\theta_{i}}\right)}{\Gamma\left(k_{i}\right)}
\end{gathered}
$$

in which $\gamma(k, x)=\int_{0}^{x} y^{k-1} e^{-y} d y$ is the lower incomplete gamma function, $i \in[1, N], k_{i} \in \mathbb{R}^{+}$is shape parameter, $\theta_{i} \in \mathbb{R}^{+}$is the scale parameter, $l_{i} \in \mathbb{R}^{+}$is location parameter applied for shifting a gamma distribution to the right of the time axis.

Applying the Laplace transform of (9) and its properties [9] for (7), it results in (11).

$$
\mathcal{L}\left\{t^{q} \times u(t)\right\}=\frac{\Gamma(q+1)}{s^{q+1}}
$$

or $\frac{t^{q}}{\Gamma(q+1)} \times u(t)=\mathcal{L}^{-1}\left\{\frac{1}{s^{q+1}}\right\}$ where $-1<q \in \mathbb{R}$

$$
=\mathcal{L}\left\{\frac{\left[t-l_{i}\right]^{\left(k_{i}-1\right)} \times e^{-\frac{\left(t-l_{i}\right)}{\theta_{i}}}}{\theta_{i}^{k_{i}} \times \Gamma\left(k_{i}\right)} \times u\left(t-l_{i}\right)\right\}=\frac{e^{-l_{i} \times s}}{\theta_{i}^{k_{i}} \times\left(s+\frac{1}{\theta_{i}}\right)^{k_{i}}}
$$

With an assumption of $\theta_{i}=\theta \forall i \in[1, N]$ and applying the Laplace transform [8], it can be rewritten from (3) and (11) as follows:

$$
\begin{aligned}
& P D F_{\Sigma}(t)=\mathcal{L}^{-1}\left\{\prod_{i=1}^{N}\left[\mathcal{L} P D F_{i}(t)\right]\right\} \\
& =\frac{1}{\theta^{\left(\sum_{i=1}^{N} k_{i}\right)}} \times \mathcal{L}^{-1}\left\{\frac{e^{-\sum_{i=1}^{N} l_{i} \times s}}{\left(s+\frac{1}{\theta}\right)^{\left(\sum_{i=1}^{N} k_{i}\right)}}\right\}= \\
& =\frac{\left[t-\sum_{i=1}^{N} l_{i}\right]^{\left(\sum_{i=1}^{N} k_{i}-1\right)} \times e^{-\frac{t-\sum_{i=1}^{N} l_{i}}{\theta}}}{\theta^{\left(\sum_{i=1}^{N} k_{i}\right)_{\times}\left(\sum_{i=1}^{N} k_{i}\right)}} \times u\left(t-\sum_{i=1}^{N} l_{i}\right)= \\
& =\frac{\left[t-l_{\Sigma}\right]^{\left(k_{\Sigma}-1\right)} \times e^{-\frac{t-l_{\Sigma}}{\theta_{\Sigma}}}}{\theta_{\Sigma}^{k_{\Sigma}} \times \Gamma\left(k_{\Sigma}\right)} \times u\left(t-l_{\Sigma}\right)
\end{aligned}
$$

in which, $k_{\Sigma}=\sum_{i=1}^{N} k_{i}, \theta_{\Sigma}=\theta$ and $l_{\Sigma}=\sum_{i=1}^{N} l_{i}$.

It can be transformed from (6) and (12) as follows:

$$
\begin{gathered}
C D F_{\Sigma}(t)=\int_{-\infty}^{t} P D F_{\Sigma}(x) d x= \\
=\int_{l_{\Sigma}}^{t} \frac{\left[x-l_{\Sigma}\right]^{\left(k_{\Sigma}-1\right)} \times e^{-\frac{\left(x-l_{\Sigma}\right)}{\theta}}}{\theta_{\Sigma}^{k_{\Sigma}} \times \Gamma\left(k_{\Sigma}\right)} d x= \\
=\frac{1}{\Gamma\left(k_{\Sigma}\right)} \times \int_{l_{\Sigma}}^{t}\left[\left(\frac{x-l_{\Sigma}}{\theta_{\Sigma}}\right)^{\left(k_{\Sigma}-1\right)} \times e^{-\left(\frac{x-l_{\Sigma}}{\theta_{\Sigma}}\right)}\right] d\left(\frac{x-l_{\Sigma}}{\theta_{\Sigma}}\right) \\
= \\
=\frac{1}{\Gamma\left(k_{\Sigma}\right)} \times \int_{0}^{\left(\frac{t-l_{\Sigma}}{\theta}\right)}\left[y^{\left(k_{\Sigma}-1\right)} \times e^{-y}\right] d y=\frac{\gamma\left(k_{\Sigma}, \frac{t-l_{\Sigma}}{\theta_{\Sigma}}\right)}{\Gamma\left(k_{\Sigma}\right)} .
\end{gathered}
$$

The composed delay distribution of (12) and (13) canalso be represented in a standardized form of scale as follows:

$$
P D F_{\Sigma}\left(t, \theta, k, \frac{k}{2}\right)=\frac{1}{\theta} \times P D F_{\Sigma}\left(t / \theta_{\Sigma}, 1, \frac{k}{2}, \frac{k}{2} / \theta_{\Sigma}\right)
$$

$C D F_{\Sigma}\left(t, \theta_{\Sigma}, k, t\right)=C D F_{\Sigma}\left(t / \theta_{\Sigma}, 1, k, \frac{k}{2} / \theta_{\Sigma}\right)$.

The characteristic measures of composed distribution of IP packet delay in an internetwork such as the mean $\left(\mu_{\Sigma}\right)$, variance $\left(\sigma_{\Sigma}^{2}\right)$ and skewness $\left(\gamma_{\Sigma}\right)$

$$
\mathcal{L}^{*}\left\{C D F_{i}(t)\right\}=\mathcal{L}\left\{P D F_{i}(t)\right\}=
$$


Research, Development and Application on Information and Communication Technology

can be computed from their definitions [10] and (12) as follows:

$$
\begin{aligned}
& \mu_{\Sigma}=\int_{0}^{+\infty}\left[t \times P D F_{\Sigma}(t)\right] d t=l_{\Sigma}+\theta_{\Sigma} \times k_{\Sigma} \\
& \sigma_{\Sigma}^{2}=\int_{0}^{+\infty}\left[\left(t-\mu_{\Sigma}\right)^{2} \times P D F_{\Sigma}(t)\right] d t=\theta_{\Sigma}^{2} \times k_{\Sigma} ;(17) \\
& \gamma_{\Sigma}=\int_{0}^{+\infty}\left[\left(\frac{t-\mu_{\Sigma}}{\sigma_{\Sigma}}\right)^{3} \times P D F_{\Sigma}(t)\right] d t=\frac{2}{\sqrt{k_{\Sigma}}}
\end{aligned}
$$

\section{B. Special Cases of the Model}

A special case is considered for a network path through $N$ sections in which IP packet delayspossess independent and identically shifted gamma distributionwith the following assumption:

$$
k_{i}=k, \theta_{i}=\theta \text { and } l_{i}=l \forall i \epsilon[1, N],
$$

in which $k \in \mathbb{R}^{+}$is the shape parameter, $\theta \in \mathbb{R}^{+}$is the scale parameter and $l \in \mathbb{R}^{+}$is the location parameter of the IP packet delay distribution in each network section. With the assumption of (19), composed distribution parameters will be resulted in (20):

$$
k_{\Sigma}=N \times k, \theta_{\Sigma}=\theta, \underline{z}=N \times l .
$$

Another special case is considered for an integer value of the composed distribution shape parameter i.e. $k_{\Sigma} \in \mathbb{N}^{+}$, in which case, the gamma function can be rewritten as $\Gamma\left(k_{\Sigma}\right)=\left(k_{\Sigma}-1\right)$ !. On the other hand, repeated application of the recurrence relation for the lower incomplete gamma function leads to the power series expansion as follows:

$$
\gamma\left(k_{\Sigma}, \frac{t-l_{\Sigma}}{\theta_{\Sigma}}\right)=\sum_{i=0}^{+\infty} \frac{e^{-\frac{t-l_{\Sigma}}{\theta_{\Sigma}}}}{k_{\Sigma} \times\left(k_{\Sigma}+1\right) \times \ldots \times\left(k_{\Sigma}+i\right)} .
$$

Therefore, theequations (12) and (13) can be rewritten as follows:

$$
\begin{gathered}
P D F_{\Sigma}(t)=\frac{\left[t-l_{\Sigma}\right]^{\left(k_{\Sigma}-1\right)} \times e^{-\frac{t-l_{\Sigma}}{\theta_{\Sigma}}}}{\theta_{\Sigma}^{k_{\Sigma}} \times\left(k_{\Sigma}-1\right) !} \times u\left(t-l_{\Sigma}\right) ; \\
C D F_{\Sigma}(t)=\sum_{i=0}^{+\infty} \frac{\left[\frac{t-l_{\Sigma}}{\theta_{\Sigma}}\right]^{\left(k_{\Sigma}+i\right)} \times e^{-\frac{t-l_{\Sigma}}{\theta_{\Sigma}}}}{\left(k_{\Sigma}-1\right) ! \times k_{\Sigma} \times\left(k_{\Sigma}+1\right) \times \ldots \times\left(k_{\Sigma}+i\right)}=
\end{gathered}
$$

$$
=\sum_{i=0}^{+\infty} \frac{\left[\frac{t-l_{\Sigma}}{\theta_{\Sigma}}\right]^{\left(k_{\Sigma}+i\right)} \times e^{-\frac{t-l_{\Sigma}}{\theta_{\Sigma}}}}{\left(k_{\Sigma}+i\right) !}=e^{-\frac{t-l_{\Sigma}}{\theta} \times \sum_{i=k_{\Sigma}}^{+\infty}\left[\frac{1}{i !} \times\right.}
$$

\section{FitnessTests of the Model}

The empirical tests based the topology of Fig. 1 have been implemented in the next generation core network of the Vietnam Post and Telecom group (VNPT), a telecommunication network operator, for verification [12]. Measurement points are located at edge and core routers. Each network section is determined by a set of link between adjacent routers. The measurement method and requirements are based on the ITU-T O.211 [13]. Pieces of information dedicated to delay measurement such as time-stamp, packet sequence number, etc., are inserted in the IP performance measurement signature (IPPMS) at the endof service data unit of IP packets. Besides, Differentiated Services Code Point (DSCP) and MPLS experimental bits (EXP) are used to differentiate IP packets for Internet traffic from others in multi-service environment. Measurement samples of different packet sizes from 80 bytes to 1500 bytes and even jumbo packet size of 9192 bytes are collected in periods of 60 minutes, 24 hours, 7 days or 1 month and at different times with average traffic load from $40 \%$ to $80 \%$ of the network capacity. More than 100 data sets with the sample size over 80000 packets have been collected during the testing time. The accuracy degree of measurement data is in millisecond.

The maximum likelihood estimation is used to find the empirical distribution parameter values which can be compared with calculated ones to result in parameter errors. Finally, a method of statistical test is used to assess the fitness of empirical distribution in accordance with a hypothetic gamma distribution as follows [1], [11], [12]:

- Step 1: Obtain the order statistics $X_{1}<X_{2}<\ldots<$ $X_{N}$ from the measured packet delay values (data)

- Step 2: Transform data by the Probability Integral Transform method $\left\{X_{i}\right\}_{i \in[1, N} \rightarrow\left\{Y_{i}=C D F\left(X_{i}, k, \theta, l\right)\right\}_{i \in[l, N]}$, in which, $k, \theta$ and 
lare estimated empirical distribution parameters and $C D F(x, k, \theta, l)$ is ahypothetic gamma cumulative distribution function. It is expected that $Y_{i}$ are uniformly independent and identically distributed on interval of $[0,1]$

- Step 3: Calculate empirical distribution function:

$$
\begin{gathered}
F_{N}(y)=\frac{1}{N} \sum_{i=1}^{N} I_{\left(Y_{i} \leq y\right)} \text { where } I_{\left(Y_{i} \leq y\right)} \\
=\left\{\begin{array}{l}
1 \text { if } Y_{i} \leq y \\
0 \text { if } Y_{i}>y^{.}
\end{array}\right.
\end{gathered}
$$

- Step 4: Statistical error percentage of the model is obtained as following:

$$
\begin{array}{r}
E(\%)=100 \times \frac{\sum_{i=1}^{N}\left|F_{N}\left(Y_{i}\right)-U\left(Y_{i}\right)\right|}{N \times E_{\max }} \\
=100 \times \frac{\sum_{i=1}^{N}\left|F_{N}\left(Y_{i}\right)-Y_{i}\right|}{N \times E_{\max }}
\end{array}
$$

In which, $E_{\max }$ is the maximum discrepancy from a true uniformdistribution $U(x)$ that may occur:

$$
E_{\text {max }}=\int_{0}^{1} \sup \{U(x), 1-U(x)\} d x
$$

The empirical results show that most of parameter errors are in the range of $4-9 \%$. The statistical errorsbetween the theoretical model and experimental samples are observed in the range of $2-5 \%$ for each network section and the range of 3-10\% for

\begin{tabular}{|c|c|c|c|c|c|c|c|c|c|c|c|}
\hline \multirow{2}{*}{$\begin{array}{l}\text { Test } \\
\text { No }\end{array}$} & \multirow[t]{2}{*}{$\mathbf{i}$} & \multirow[t]{2}{*}{$\mathbf{k}_{\mathrm{i}}$} & \multirow[t]{2}{*}{$\theta_{\mathrm{i}}$} & \multirow[t]{2}{*}{$\mathbf{l}_{\mathbf{i}}$} & \multicolumn{3}{|c|}{\begin{tabular}{|c|} 
Calculated \\
Parameter Values
\end{tabular}} & \multicolumn{3}{|c|}{$\begin{array}{c}\text { EmpiricalParam } \\
\text { eter Values }\end{array}$} & \multirow{2}{*}{$-\begin{array}{c}\mathbf{E} \\
(\%)\end{array}$} \\
\hline & & & & & $\mathbf{k}_{\Sigma}$ & $\theta_{\Sigma}$ & $\mathbf{I}_{\Sigma}$ & $\mathbf{k}_{\Sigma}^{\prime}$ & $\theta_{\Sigma}^{\prime}$ & $\mathbf{l}_{\Sigma}^{\prime}$ & \\
\hline \multirow{4}{*}{ I } & 1 & 0.1 & 3.5 & 00.7 & \multirow{4}{*}{1.1} & \multirow{4}{*}{3.5} & \multirow{4}{*}{3.0} & \multirow{4}{*}{1.2} & \multirow{4}{*}{3.3} & \multirow{4}{*}{3.1} & \multirow{4}{*}{5.0} \\
\hline & 2 & 0.2 & 3.5 & 00.8 & & & & & & & \\
\hline & 3 & 0.5 & 3.5 & 00.9 & & & & & & & \\
\hline & 4 & 0.3 & 3.5 & 00.6 & & & & & & & \\
\hline \multirow{4}{*}{ II } & 1 & 0.9 & 3.0 & 05.0 & \multirow{4}{*}{19.2} & \multirow{4}{*}{3.0} & \multirow{4}{*}{50.8} & \multirow{4}{*}{19.9} & \multirow{4}{*}{2.8} & \multirow{4}{*}{52.9} & \multirow{4}{*}{4.8} \\
\hline & 2 & 9.4 & 3.0 & 20.5 & & & & & & & \\
\hline & 3 & 3.8 & 3.0 & 10.1 & & & & & & & \\
\hline & 4 & 5.1 & 3.0 & 15.2 & & & & & & & \\
\hline \multirow{3}{*}{ III } & 1 & 0.1 & 5.0 & 03.3 & \multirow{3}{*}{1.9} & \multirow{3}{*}{5.0} & \multirow{3}{*}{24.6} & \multirow{3}{*}{1.8} & \multirow{3}{*}{4.8} & \multirow{3}{*}{25.8} & \multirow{3}{*}{5.1} \\
\hline & 2 & 1.0 & 5.0 & 09.0 & & & & & & & \\
\hline & 3 & 0.3 & 5.0 & 05.2 & & & & & & & \\
\hline
\end{tabular}
internetworks. Parameter and model errorsdepend on

\begin{tabular}{|c|c|c|c|c|c|c|c|c|c|c|c|}
\hline & 4 & 0.5 & 5.0 & 07.1 & & & & & & & \\
\hline \multirow{3}{*}{ IV } & 1 & 4.6 & 1.0 & 08.9 & \multirow{3}{*}{10.6} & \multirow{3}{*}{1.0} & \multirow{3}{*}{20.7} & \multirow{3}{*}{11.1} & \multirow{3}{*}{0.9} & \multirow{3}{*}{21.5} & \\
\hline & 2 & 2.2 & 1.0 & 05.6 & & & & & & & 5.5 \\
\hline & 3 & 3.8 & 1.0 & 06.2 & & & & & & & \\
\hline \multirow{3}{*}{$\mathrm{V}$} & 1 & 0.5 & 4.3 & 03.5 & \multirow{3}{*}{4.5} & \multirow{3}{*}{4.3} & \multirow{3}{*}{10.7} & \multirow{3}{*}{4.2} & \multirow{3}{*}{4.1} & \multirow{3}{*}{11.1} & \\
\hline & 2 & 2.7 & 4.3 & 04.8 & & & & & & & 3.5 \\
\hline & 3 & 1.3 & 4.3 & 02.4 & & & & & & & \\
\hline
\end{tabular}
traffic load condition, bandwidth or capacity and network scope. Several typical results are described in Table 1, Figure 2 and Figure 3.

\section{Table 1. Fitness Tests of The Model}

\begin{tabular}{|c|c|c|c|c|c|c|c|c|c|c|}
\hline Case & $\mathbf{N}$ & $\mathbf{k}_{\mathrm{i}}$ & $\theta_{\mathrm{i}}$ & $\mathbf{l}_{\mathrm{i}}$ & $\mathbf{k}_{\Sigma}$ & $\theta_{\Sigma}$ & $l_{\Sigma}$ & $\boldsymbol{\mu}_{\Sigma}$ & $\sigma_{\Sigma}^{2}$ & $\gamma_{\Sigma}$ \\
\hline \multirow{5}{*}{01} & \begin{tabular}{ll|}
1 \\
\end{tabular} & 0.5 & 0.5 & 05 & 0.5 & 0.5 & 5 & 5.25 & 0.125 & 2.828 \\
\hline & 2 & 0.5 & 0.5 & 05 & 1.0 & 0.5 & 10 & 10.50 & 0.250 & 2.000 \\
\hline & 3 & 0.5 & 0.5 & 05 & 1.5 & 0.5 & 15 & 15.75 & 0.375 & 1.633 \\
\hline & \begin{tabular}{|l|}
4 \\
\end{tabular} & 0.5 & 0.5 & 05 & 2.0 & 0.5 & 20 & 21.00 & 0.500 & 1.414 \\
\hline & \begin{tabular}{|l|}
5 \\
\end{tabular} & 0.5 & 0.5 & 05 & 2.5 & 0.5 & 25 & 26.25 & 0.625 & 1.265 \\
\hline \multirow{5}{*}{02} & 3 & 0.3 & 1.0 & 08 & 0.9 & 1.0 & 24 & 24.90 & 0.900 & 2.108 \\
\hline & \begin{tabular}{|l|}
3 \\
\end{tabular} & 0.5 & 1.0 & 08 & 1.5 & 1.0 & 24 & 25.50 & 1.500 & 1.633 \\
\hline & 3 & 1.5 & 1.0 & 08 & 4.5 & 1.0 & 24 & 28.50 & 4.500 & \begin{tabular}{|l|}
0.943 \\
\end{tabular} \\
\hline & 3 & 3.0 & 1.0 & 08 & 9.0 & 1.0 & 24 & 33.00 & 9.000 & \begin{tabular}{|l}
0.667 \\
\end{tabular} \\
\hline & 3 & 5.0 & 1.0 & 08 & 15 & 1.0 & 24 & 39.00 & 15.00 & 0.667 \\
\hline \multirow{5}{*}{03} & 3 & 2.0 & 1.0 & 10 & 6.0 & 1.0 & 30 & 36.00 & 6.000 & 0.516 \\
\hline & \begin{tabular}{|l|}
3 \\
\end{tabular} & 2.0 & 2.0 & 10 & 6.0 & 2.0 & 30 & 42.00 & 24.00 & \begin{tabular}{|l|}
0.816 \\
\end{tabular} \\
\hline & 3 & 2.0 & 3.0 & 10 & 6.0 & 3.0 & 30 & 48.00 & 54.00 & 0.816 \\
\hline & 3 & 2.0 & 4.0 & 10 & 6.0 & 4.0 & 30 & 54.00 & 96.00 & 0.816 \\
\hline & 3 & 2.0 & 5.0 & 10 & 6.0 & 5.0 & 30 & 60.00 & 150.0 & 0.816 \\
\hline \multirow{5}{*}{04} & 3 & 2.5 & 6.0 & 05 & 7.5 & 6.0 & 15 & 60.00 & 270.0 & 0.816 \\
\hline & 3 & 2.5 & 6.0 & 10 & 7.5 & 6.0 & 30 & 75.00 & 270.0 & \begin{tabular}{|l|l}
0.730 \\
\end{tabular} \\
\hline & 3 & 2.5 & 6.0 & 15 & 7.5 & 6.0 & 45 & 90.00 & 270.0 & 0.730 \\
\hline & 3 & 2.5 & 6.0 & 20 & 7.5 & 6.0 & 60 & 105.0 & 270.0 & 0.730 \\
\hline & 3 & 2.5 & 6.0 & 25 & 7.5 & 6.0 & 75 & 120.0 & 270.0 & 0.730 \\
\hline \multirow{4}{*}{05} & \begin{tabular}{ll|}
1 \\
\end{tabular} & 0.9 & 3.0 & 05 & \multirow{4}{*}{19.2} & \multirow{4}{*}{3.0} & \multirow{4}{*}{50} & \multirow{4}{*}{107.6} & \multirow{4}{*}{172.8} & \multirow{4}{*}{0.456} \\
\hline & \begin{tabular}{ll|} 
\\
\end{tabular} & 3.8 & 3.0 & 10 & & & & & & \\
\hline & 1 & 5.1 & 3.0 & 15 & & & & & & \\
\hline & 1 & 9.4 & 3.0 & 20 & & & & & & \\
\hline \multirow{4}{*}{06} & 1 & 0.1 & 5.0 & 03 & \multirow{4}{*}{1.9} & \multirow{4}{*}{5.0} & \multirow{4}{*}{24} & \multirow{4}{*}{33.50} & \multirow{4}{*}{167.5} & \multirow{4}{*}{1.451} \\
\hline & 1 & 0.3 & 5.0 & 05 & & & & & & \\
\hline & 1 & 0.5 & 5.0 & 07 & & & & & & \\
\hline & 1 & 1.0 & 5.0 & 09 & & & & & & \\
\hline
\end{tabular}

Note: the unit of $l_{i}, l_{\Sigma}$ and $l_{\Sigma}{ }^{\prime}$ is millisecond.

Table 2. Variants of The Model in Accordance with Distribution Parameters Variation

\section{Model variants and parameter relationship}

Different variants of the composed model in accordance with parameter variation of component distributions can be derived from the general model of (12) and (13). Some specific and typical cases are summarized and compared in Table II and figures from number 4 to number 9 , in which $N$ is the number of network sections; $k_{i}, \theta_{i}, l_{i}$ are the shape, scale, location parameters respectively of component delay while $k_{\Sigma}, \theta_{\Sigma}, l_{\Sigma}$ are those of composed delay; $\mu_{\Sigma}$ is mean and $\sigma_{\Sigma}^{2}$ is variant of the composed distribution.

There are six case studies, each of them corresponding to the variation of one or more parameters. The distribution parameters of IP packet 
Research, Development and Application on Information and Communication Technology
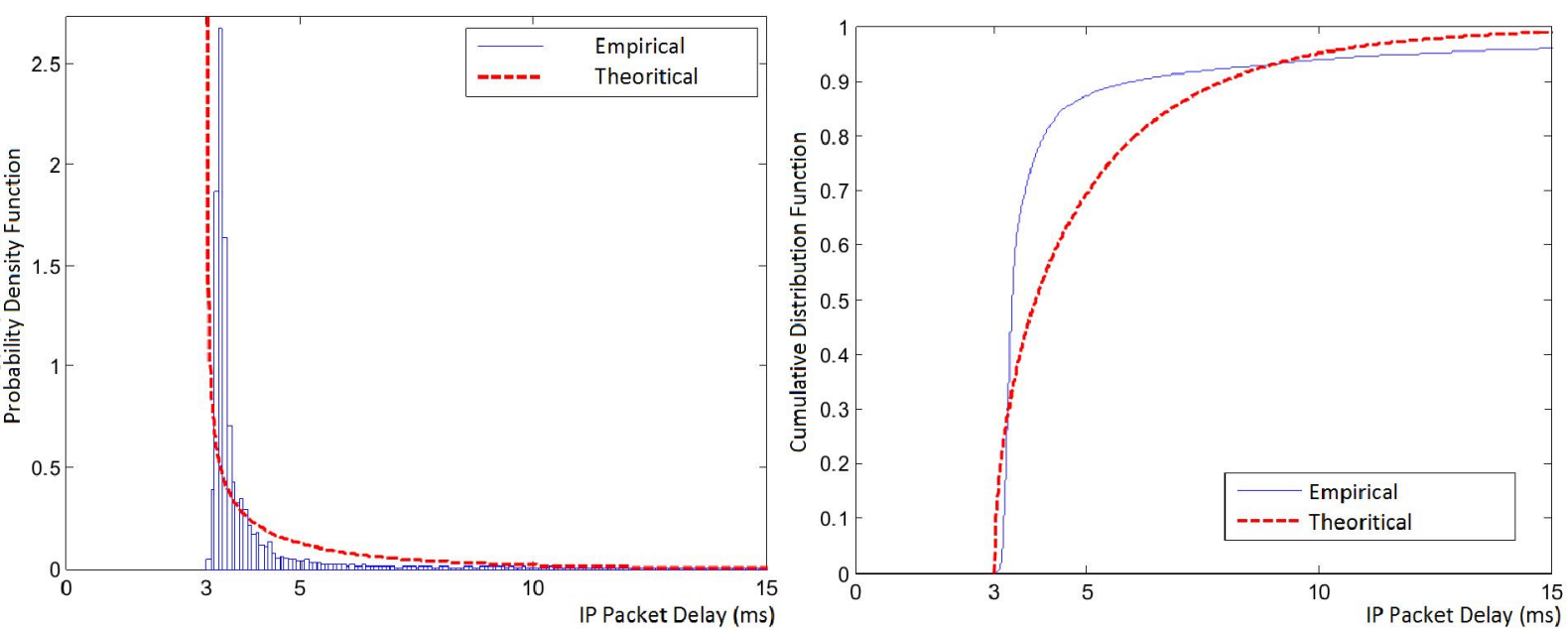

Figure 2. A comparision of theoretical and experimental IP packet delay of shifted gamma distribution: Test Number I
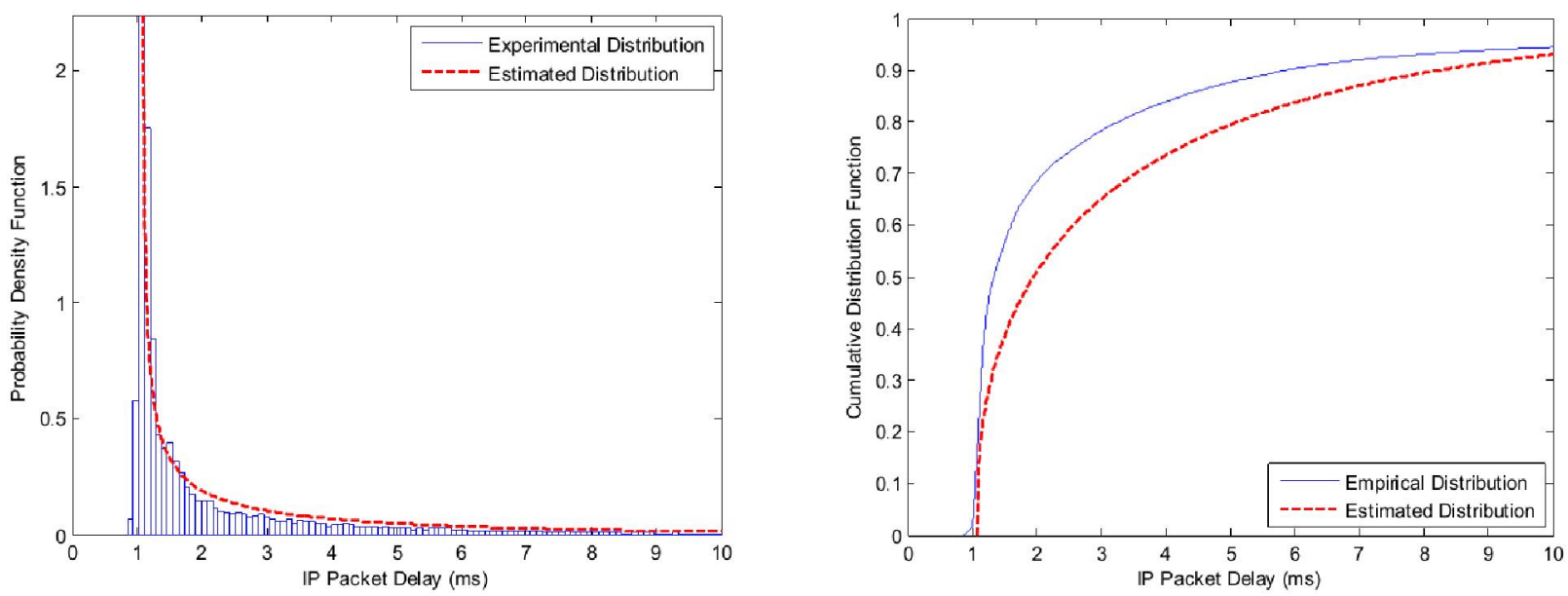

Figure 3. A comparision of theoretical and experimental IP packet delay of shifted gamma distribution: Test Number $V$

delay in $N$ network sections are identical for the first 4 case studies and they are different for the remainders $\left(5^{\text {th }}\right.$ and $\left.6^{\text {th }}\right)$. The distributions of IP packet delay over the whole network are composed for a varied number of network sections in the first case (Figure 4), varied values of component location parameters in the second case (Fig.5), varied values of component scale parameters in the third case (Fig.6), varied values of component shape parameters in the fourth case (Fig.7). Finally, the composed and component distributions corresponding to different value sets of their parameters are demonstrated in the remainders (Fig. 8 and Fig. 9).

\section{EVALUATION AND DISCUSSION}

It is realized in the paper that the convolution and Laplace transform can be used to compose IP packet delay distribution in the whole internetwork from component ones. The problem has been resolved completely with the assumption of independent shifted gamma distributions of IP packet delay in different network sections.

Most of the experimental results show that Internet traffic packet delay in next generation core network sections as well as over the whole internetworks can be approximately fitted by a shifted gamma 


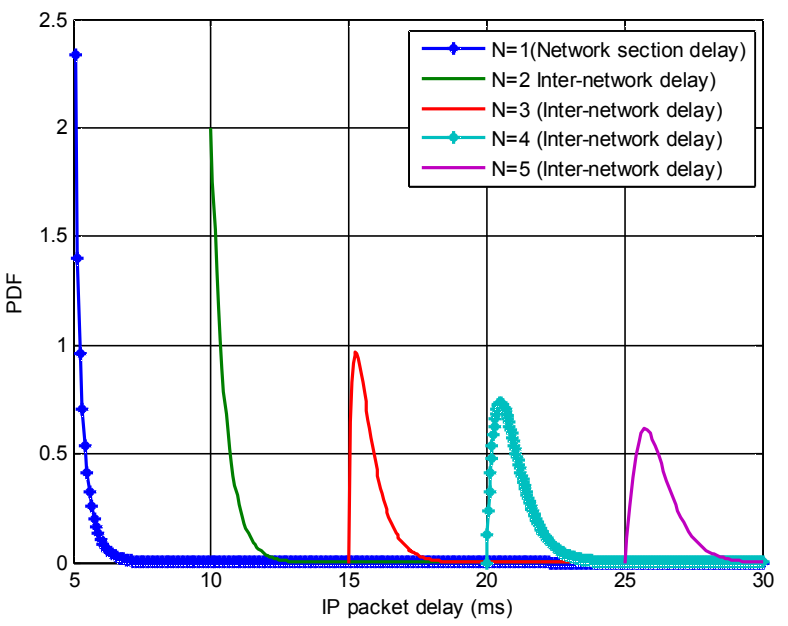

(a). Probability density function

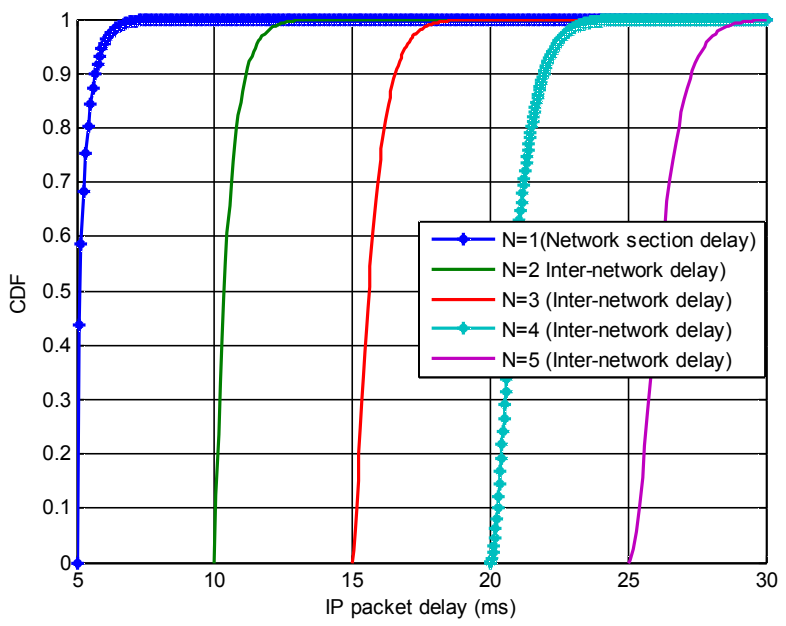

(b). Cumulative distribution function

Figure 6. Composed distributions of IP packet in an internetwork composed of varied number of network sections (Case 01)

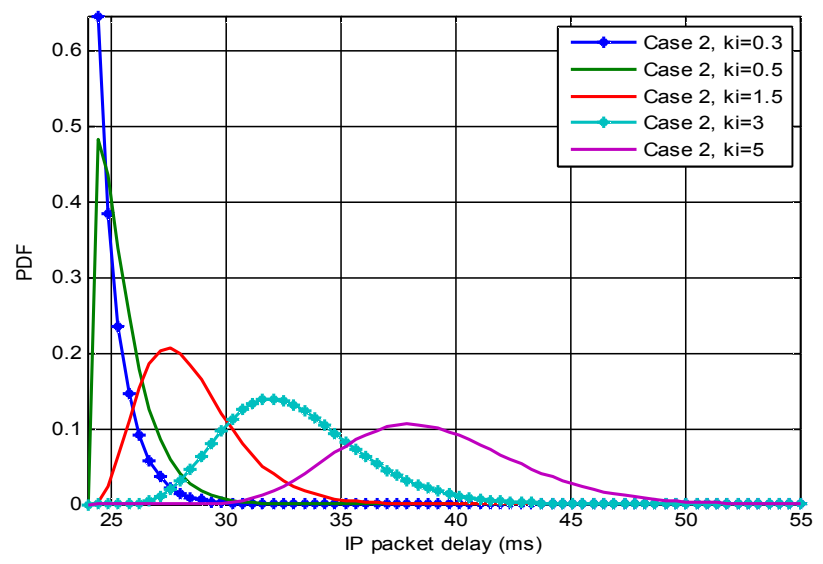

(a). Probability density function

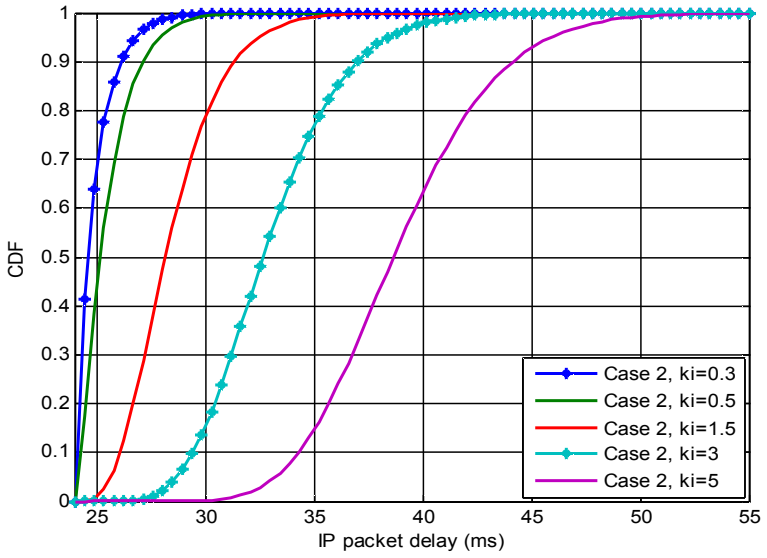

(b). Cumulative distribution function

Figure 7. Composed distributions of internetwork delay for varied values of distribution shape parameters in network sections (Case 02)

distribution model with model error within the range of $3-10 \%$.

With an introduction of shifted gamma distribution family denoted by $\Gamma^{\prime}(k, \theta, l)$ in which $k, \theta$ and $l$ are shape, scale and location parameter respectively, it can be deduced from the composed model that if IP packet delay of $D_{i}$ in each network section satisfies $D_{i}$ $\sim \Gamma^{\prime}\left(k_{i}, \theta, l_{i}\right) \forall i \in[1, N]$, then its composed distribution in the whole internetwork conforms $D_{\Sigma} \sim \Gamma^{\prime}\left[\left(k_{\Sigma}=\right.\right.$
$\left.\left.\sum_{i=1}^{N} k_{i}\right), \theta_{\Sigma},\left(k_{2}=\sum_{i=1}^{N} l_{i}\right)\right]$, in which the composed shape and location parameter equal to sums of component ones while scale parameters is invariant. The support of composed distribution is defined in the range of $\left[l_{\Sigma},+\infty\right)$. It can be reasoned that the appearance of location parameters or shifting property of IP packet delay distribution originates from the nature of its lower limit in real networks. 
Research, Development and Application on Information and Communication Technology

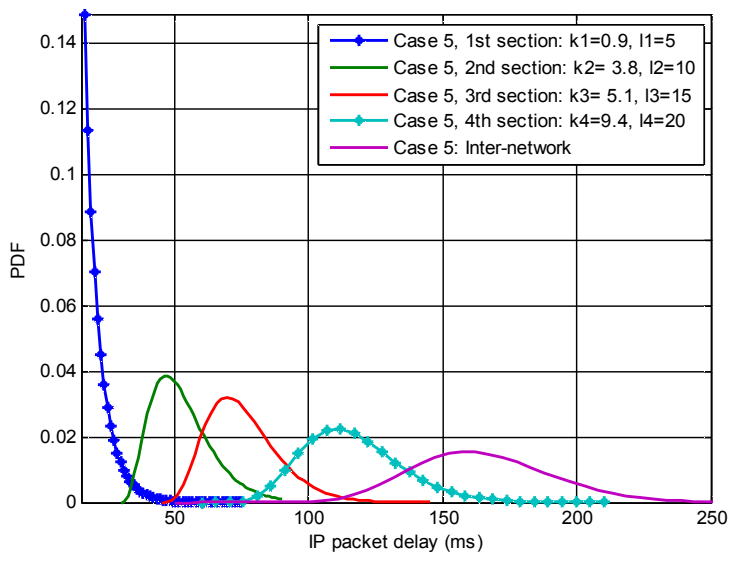

((a). Probability density function

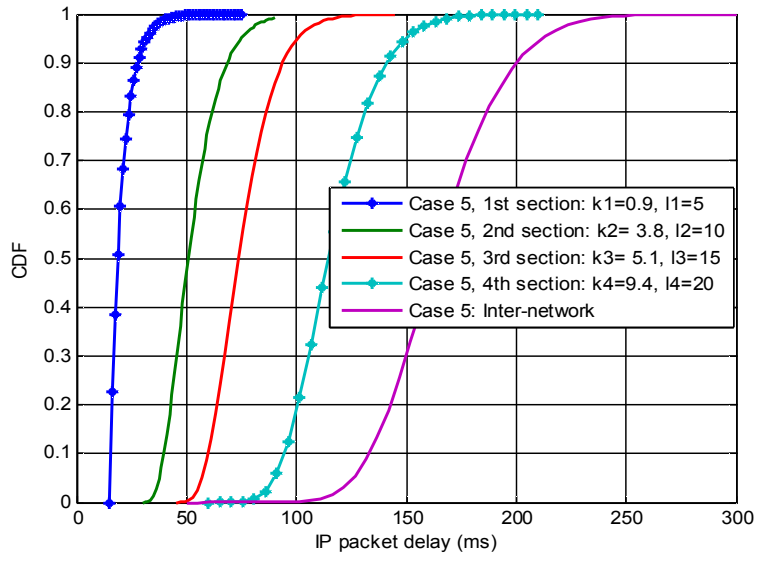

(b). Cumulative distribution function

Figure 8. Network sections and internetwork delay distributions with different sets of parameter values (Case 05)

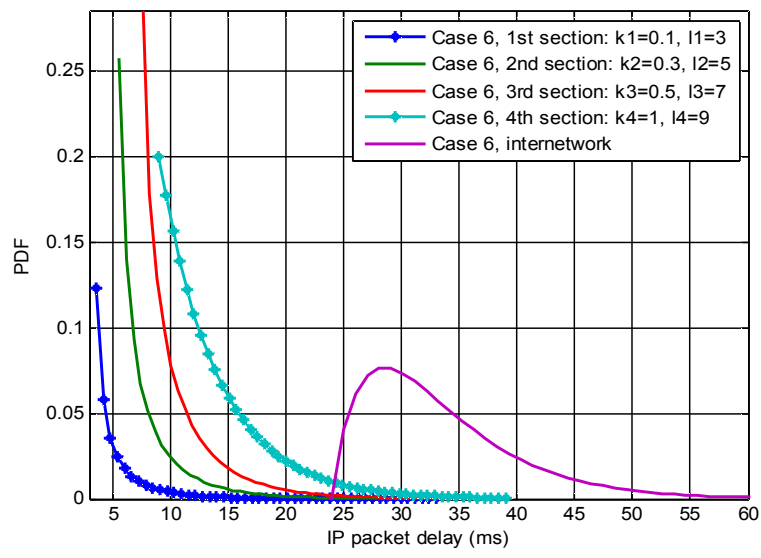

(a). Probability density function

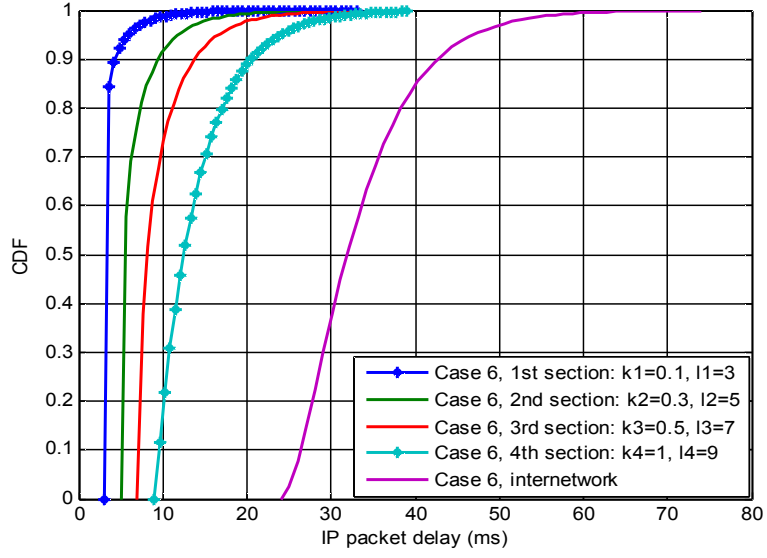

(b). Cumulative distribution function

Figure 9. Network sections and internetwork delay distributions with different sets of parameter values (Case 06)

In the case of homogeneous delay distribution, it can be observed that shape and location parameter of composed delay distribution is $N$ times larger than that of component ones.

It can be recognized from the mathematical model as well as from the data samples that variations of these parameters have different effects on IP packet delay distribution. The value of $\theta_{\Sigma}$ determines statistical dispersion of delay distribution as in Fig. 10: larger value causes distribution more spread outalong the time axis toward $+\infty$ while lower probability density, andvice versa (the $3^{\text {rd }}$ case study). The value of $l_{\Sigma}$ determines the location or shift of the distribution along the time axis. Larger location value causes distribution shifted more to the right and vice versa (the $4^{\text {th }}, 5^{\text {th }}$ and $6^{\text {th }}$ case studies). The value of $k_{\Sigma}$ affects distribution shape rather than simply 
shifting it as the location parameter does or stretching/shrinking it as the scale parameter does as described in Fig. 9, Fig. 12 and Fig. 13 (the $2^{\text {nd }}, 5^{\text {th }}$ and $6^{\text {th }}$ case studies). - It can be seen two variant forms of distribution corresponding to shape parameter value lager or lower than 1 .

Regarding the relation between lower moments of composed distribution and parametersof component one, it is observed that the skewness of composed distribution depends only on the shape parameter yalues of component ones, its variance depends on boththeir shape and scale parameter values while its mean dependson their all parameters values.

Finally, acomposed distribution model can be represented more simply or explicitly in mathematics for several special cases such as identical distributions of IP packet delay in network sections or integer values of shape parameters. In the second case, it becomes a kind of shifted Erlang distribution.

\section{CONCLUSION}

It is proved in the paper that Internet packet delay distribution in the next generation multi-service core networks can be modeledby a shifted gamma distribution.

Moreover, delay distribution of IP packet transported over an internetwork can also be composed from component ones. A complete mathematical model of shifted gamma distribution for estimatingIP packet delay over an internetwork from component ones has been established and proposed as a new result in the paper.

The above method and model are based on convolution, Laplace transform and other mathematic basics with certain hypotheses. The model has also been simplified for special cases of homogeneous networks or an integer value of composed distribution shape parameter.

Typical case studies of the model have also been analyzed, compared and evaluated to summarize qualitative and quantitative characteristics of IP packet delay distributions and to expose the model properties and their parameters relationship as a new judgment. The composed distribution inherits properties from component ones in the sense that they belong to the same family of shifted gamma distribution with the additive property of shape and location parameters. Different parameters of component delay distributions have different effects and contributions on the composed one. There are two variants forms of distribution depending on shape parameter value. They are stretched or shrank according to scale parameter and shifted according to location one. The mean value of a composed distribution depends on shape, scale and location parameters of component ones, its variance depends on shape and scale parameters and finally its skewness depends only on shape parameters.

The research results in the paper can be applied in analyzing and evaluating IP packet delay over core network sections as well as composing delay over internetworks. It is especially useful in cases of an internetwork spreading over different provider networks, in which delay metrics are normally collected independently in each separated network section but not over the whole one, whereas detailed measure-ment data are seldom be shared among operators. Moreover, fewer segment measurement results used in calculating thousands of concatenated network sections will help minimize much cost and overhead. The results can be used as one of the basics for developing traffic engineering in IP core networks with the goal of minimizing delay in order to improve the network performance and quality of services.

Other studies are needed for the cases of mutually dependent distributions, disparate values of the scale parameters or other kinds of distributions matched in different networks. Besides, additional methods should be developed for mixed distributions or ones that are not well defined by explicit mathematical functions.

\section{REFERENCES}

[1] Adrian Popescu and Doru Constantinescu (2005), Measurement of One-Way Transit Time in IP Routers, Blekinge Institute of Technology, Sweden.

[2] Paxson V. (1997), Measurements and Analysis of Endto-End Internet Dynamics, PhD Dissertation, University of California at Berkeley, 1997.

[3] ITU-T (2011), Network Performance Objectives for IPbased Services, Rec. Y.1541, 2011. 
Research, Development and Application on Information and Communication Technology

[4] A. Mukherjee (1994), On the Dynamics and Significance ofLow Frequency Components of Internet Load, Internetworking: Research and Experience, Vol. 5, No. 4, pp. 3-4 , 22-29,45, 1994.

[5] Ana Hernandez, Eduardo Magana (2007), One-way Delay Measurement and Characterization, Proceedings of the IEEE ICNS '07, Washington, DC, USA, 2007, pp.4.

[6] Bovy C. J., Mertodimedjo H. T., Hooghiemstra G., Uijterwaal H. and Van Mieghem P. (2002), Analysis of End-to-End Delay Measurements in Internet, ACM PAM, Fort Collins, Colorado, USA, 2002.

[7] D. Constantinescu and A. Popescu (2006), Modeling of One-Way Transit Time in IP Routers, Proceedings of the Advanced Int'l Conference on Telecommunications and Int'l Conference on Internet and Web Applications and Services AICT-ICIW '06, February 2006.

[8] Trivedi K.S (1982), Probability and Statistics with Reliability, Queuing, and Computer Science Applications, Publisher-John Wiley \& Sons, 1982.

[9] Abramowitz (1972), M. and Stegun, Handbook of Mathematical Functions with Formulas, Graphs, and Mathematical Tables, 9th printing, New York: Dover, 1972.

[10] http://mathworld.wolfram.com

[11] D'Agostino R.B. and Stephens M.A. (1986), Goodness-of-Fit Techniques, Marcel Dekker, New York.

[12] ITU-T Rec. O.211 (2006), Test and measurement equipment to perform tests at the IP layer.

\section{AUTHORS' BIOGRAPHIES}

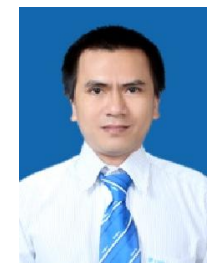

Dao Ngoc Lam was born in 1971, received M.Eng degree in Electronics and Telecommunications from the Posts and Telecommunications Institute of Technology (PTIT), Vietnamin 2007, is now a Ph.D student at PTIT. Working office: Vietnam Telecom National, VNPT.

Research interests: IP network performance, next generation network, IP traffic engineering.

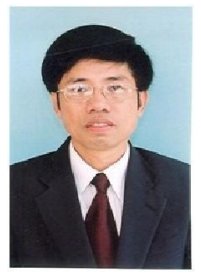

Le Huu Lap was born in 1954, received the B.Eng degree and Ph.D degree in Electronicsand Telecommunications from the Ilmenau University, Germany in 1984 and 1988. At thepresent, he is an Associate Professor and the Vice President of Posts and Telecommunications Institute of Technology (PTIT), Hanoi, Vietnam. Research interests: next generation network.

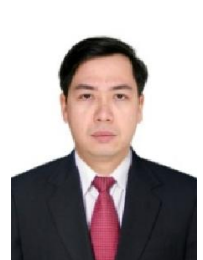

Le Nhat Thang was born in 1973, received the B.Eng degree in Radio Electronics and Communication from Hanoi University of Science and Technology (HUST), Vietnam, in 1995, the M.Eng degree in Telecom from Asian Institute of Technology (AIT), Thailand, in 2000 and $\mathrm{Ph} . \mathrm{D}$. degree in Information and Communication Technology (ICT) from theD epartment of Computer Science and Telecom (DIT), University of Trento, Italy in 2006.

$\mathrm{He}$ is currently an Associate Professor and the Head Department of Switching Engineering, Faculty of Telecom 1, PTIT, Hanoi, Vietnam. Research interests: performance analysis, modeling and simulations, trafficengineering, queueing theory and applications, QoS, routing and switching techniques, nextgeneration network. 